\title{
Surgical injury and repair of hip external rotators in THA via posterior approach: a three-dimensional MRI-evident quantitative prospective study
}

Ting Wang $^{\dagger}$, Long Shao ${ }^{\dagger}$, Wei Xu, Feilong Li and Wei Huang ${ }^{*}$

\begin{abstract}
Background: As one of the classical total hip arthroplasty (THA) approaches, the posterior approach is widely used. However, there is a lack of in-depth quantitative researches on the surgical-related injury to the hip external rotators. The purpose of this study is to quantificationally analyse the surgical injury of hip external rotators after posterior THA and explore the effect of the muscle repair on the muscle recovery using the MRI three-dimensional reconstruction technique combined with the clinical assessment.

Methods: Sixty five patients were eligible to receive a unilateral cementless THA via the posterior approach. During operation, the piriformis tendon was reattached but it was not applicable for the internal obturator muscle. We performed three-dimensional MRI reconstruction of bilateral piriformis and internal obturator muscle along with clinical assessment preoperatively, 6, 12 and 52 weeks postoperatively.

Results: Bilateral piriformis and internal obturator muscle were homogeneous preoperatively. Compared with the contralateral side, the volume atrophy and fat-muscle ratio of the piriformis on the operated side increased inconspicuously by $1.64 \%, 0.26 \%(p=0.062, p=0.071)$ at 6 weeks and $1.33 \%, 0.20 \%(p=0.057, p=0.058)$ at 12 weeks, while $7.28 \%, 2.09 \%$ and $15.71 \%, 5.14 \%$ for the internal obturator muscle $(p<0.01)$. Up to 52 weeks, the pirformis also showed significant muscle atrophy as well as fatty infiltration (increased by $7.79 \%, 4.21 \% ; p<0.01$ ), and $24.18 \%, 11.91 \%$ for the internal obturator muscle $(p<0.01)$.

Conclusion: The THA via posterior approach significantly harms the hip external rotators and the early hip external rotation function. The effective repair could be conducive to the early postoperative recovery of the hip external rotators

Trail registration: The study has been registered in Chinese Clinical Trial Registry (ChiCTR) before the clical trial started, the Clinical Trial Registry Number is ChiCTR-IOR-17013007. Registered 17 October 2017. The Trial registration is prospective registration.
\end{abstract}

Keywords: Total hip arthroplasty, External rotators, Three-dimensional MRI reconstruction, Injury, Repair

\footnotetext{
* Correspondence: huangwei68@263.net

†Ting Wang and Long Shao contributed equally to this work.

Department of Orthopedics, The First Affiliated Hospital of Chongqing

Medical University, No.1 Youyi Road, Yuan jiagang, Yuzhong District,

Chongqing, China
}

(c) The Author(s). 2019 Open Access This article is distributed under the terms of the Creative Commons Attribution 4.0 International License (http://creativecommons.org/licenses/by/4.0/), which permits unrestricted use, distribution, and reproduction in any medium, provided you give appropriate credit to the original author(s) and the source, provide a link to the Creative Commons license, and indicate if changes were made. The Creative Commons Public Domain Dedication waiver (http://creativecommons.org/publicdomain/zero/1.0/) applies to the data made available in this article, unless otherwise stated. 


\section{Background}

Total hip arthroplasty (THA) is one of the most successful orthopedic surgery in the twentieth century; it is the preferred treatment for end-stage coxarthropathy. The posterior approach, also known as the "Kocher-Langenbeck approach", is the most frequently used among multitudinous THA approaches $[1,2]$. Concerning this approach, a widespread controversy has been always existed over the surgical injury of the hip external rotators and whether to repair them or not.

Various studies have confirmed the muscle damage to the short external rotators via posterior THA from cadaveric tests, electromyography (EMG), biochemical serum markers and gait analysis [3-6]. However, there was still a lack of consensus on the extent of the muscle damage and the necessity of the muscle repair. With the development of imaging technology, magnetic resonance imaging (MRI) has been the gold standard for muscles evaluation, especially the muscular morphorlogical changes $[7,8]$. Nevertheless, to the authors' knowledge, that of the hip external rotators was barely mentioned. In addition, all of these studies were based on the two-dimensional MRI single layer analysis so that the results lacked precision and objectiveness [9].

Given all this, the aim of this study was to address the following questions using the MRI three-dimensional reconstruction technique in combination with the clinical assessment: 1) What were the changes in the muscle morphology of hip external rotators and the external rotation function after posterior THA? 2) Whether the repair of external rotators contributed to a better amelioration in the muscle morphology postoperatively?

\section{Methods}

\section{Patients}

This prospective study has been approved by Institutional Review Board and registered in Chinese Clinical Trial Registry (ChiCTR) before the clical trial started. The methods were carried out in accordance with the relevant guidelines and regulations. In this study, 127 patients prepared for unilateral THA were enrolled continuously from January 2018 to June 2018 including 74 males and 53 females. Each participant has signed the informed consent, and all were over 16 years old. Inclusion criteria comprises admission diagnosis for the development of hip dysplasia (Crowe type I, type II), avascular necrosis of the femoral head, primary hip osteoarthritis, femoral head epiphyseal Ischemic necrosis (Legg-Calve-Perthes disease). Exclusion criteria consists of contralateral hip disease or surgical history, joint ankylosis or stiffness, severe developmental hip dysplasia (Crowe type III, type IV), femoral neck fracture, intertrochanteric fractures, suppurative coxarthritis, rheumatoid arthritis, femoral or acetabular osteotomy, ipsilateral surgical history, severe systemic infection or tumor diseases, severe medical diseases, muscle weakness, muscle dystrophy or muscle atrophy related diseases, physical disability or mental illness. At last, 56 patients were eligible for the final analysis in this study (Fig. 1). Demographic data were registered (Table 1).

\section{Surgical intervention}

Each posterior THA was performed by the same senior medical practitioner. The prosthesis for each patient were all biological type. The material of femoral stem (LCU or Ribbed) and acetabular cup (T.O.P or Combi cup) were titanium alloy. In addition, the acetabular lining was high-crosslinked polyethylene and the femoral head was ceramic. During operation, the tendon insertion of the short external rotators group including piriformis, internal obturator muscle, superior gemellus, and inferior gemellus were cut off; the posterior joint capsule was cut through with a flap-shaped incision. Only the tendon of the piriformis in combination with the posterior joint capsule was non-in-situ reattached through a suture hole on the posterior part of femoral great trochanter using the non-absorbable suture (Ethibond) (Fig. 2). The same postoperative rehabilitation protocol was followed by each patient.

127 patients registered

initially to receive primary

unilateral cementless THA

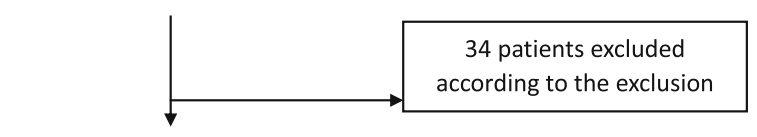

93 patients enrolled to

receive preoperative clinical investigation

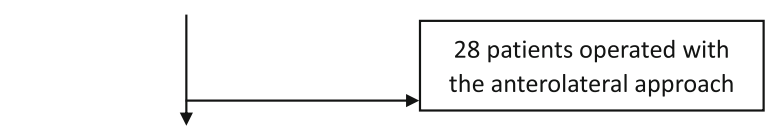

65 patients included in the study to complete the follow-up

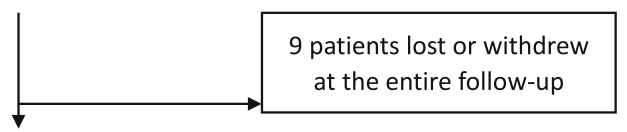

56 patients in analysis

Fig. 1 Flow chart showing screening of patients 
Table 1 Demographic Data

\begin{tabular}{ll}
\hline Demographic & \\
\hline No. of patients & 56 \\
Age(yrs) & $56.77 \pm 15.44$ \\
Gender & \\
$\quad$ Male & $33(58.92 \%)$ \\
$\quad$ Female & $23(41.08 \%)$ \\
Height(cm) & $164.05 \pm 6.47$ \\
Weight(kg) & $65.66 \pm 10.08$ \\
BMI(kg/m $\left.{ }^{2}\right)$ & $24.4 \pm 3.8$ \\
Surgical side & \\
$\quad$ Left & $32(57.14 \%)$ \\
Right & $24(42.86 \%)$ \\
Operative time(min) & $89.88 \pm 14.75$ \\
Blood loss(ml) & $145.54 \pm 66.93$ \\
Incision length(cm) & $9.16 \pm 0.83$ \\
Diagnosis & \\
ANFH & $29(51.79 \%)$ \\
DDH & $22(39.28 \%)$ \\
OA & $3(5.35 \%)$ \\
Legg-Calve-Perthes & $2(3.58 \%)$ \\
\hline BMI Body Mass & \\
\hline
\end{tabular}

BMI Body Mass Index, ANFH Avascular Necrosis of Femoral Head, DDH Developmental Dysplasia of Hip, OA Osteoarthritis

\section{Three-dimensional MRI reconstruction technique}

MRI was performed on a $1.5 \mathrm{~T}$ scanner (Signa HDxt, GE MEDICAL SYSTEMS, USA) according to a standard protocol preoperatively, 6, 12 and 52 weeks postoperatively. Images were acquired in 4-mm slices using a flexible phased-array coil and clinically established MRI sequences at radiology department. T1-weighted fast spin-echo (FSE) images were obtained using configured scan parameters $(\mathrm{TE}=$ Min Full; $\mathrm{TR}=760 \mathrm{~ms}$; matrix size $=512 * 512$; bandwidth $=31.25 \mathrm{kHz}$; field of view $=480 \mathrm{~mm}$; slice thickness $=3 \mathrm{~mm}$; spacing $=1 \mathrm{~mm}$ ). T2-weighted fast recovery with fast spin-echo (FRFSE) sequence was also acquired (Auto TR, $2820 \mathrm{~ms}$; Echo Train Length (18 mm); matrix size, $512 * 512$; bandwidth, 31.25
$\mathrm{kHz}$; field of view, $480 \mathrm{~mm}$; Freq, $228 \mathrm{kHz}$; slice thickness, $0.5 \mathrm{~mm}$; spacing, $1 \mathrm{~mm}$ ). The use of cross sections established the first cut from the level of anterior superior iliac spine to the middle of femoral shaft in each patient. The DICOM (Digital Imaging and Communications in Medicine) data of all the 56 bilateral hip joint MRI images were saved as DVD and loaded into Mimics 17.0 (Materialise, Belgium). Two researchers independently identified the contours of the bilateral external rotators using the LiveWare tool. If controversy existed over the identification between two researchers, the third professional veteran musculoskeletal radiologist with ten years of experience would make the final determination. The range of the reconstruction included all sections of the target muscle that can be clearly recognized on the cross-section. Due to the influence of muscle atrophy, the number of bilateral sections can vary. The Cronbach's Alpha and intraclass correlation coefficient for consistency between the two judgers on each thresholding value selection for all included MRI images was 0.992 and 0.983 (95\% CI $=0.972-$ $0.990, \mathrm{~F}=120.026, p=0.000)$, respectively. After selection of adipose thresholding, "negative value" operation was applied in the course of the adipose tissue reconstruction in order to ensure that the range of the two masks was exactly the same. The adipose tissue was separated from the muscle through the Boolean operation. The volume of muscle, adipose tissue and the corresponding fat-muscle ratio were calculated from the polygonal surfaces on the basis of the three-dimensional reconstruction technique (Fig. 3).

\section{Clinical assessment}

Bilateral hip external rotation range measurement was conducted on each patient by the same medical practitioner preoperatively, 6, 12 and 52 weeks postoperatively. The medical practitioner was blinded to the surgical procedure and the MRI results.

\section{Statistical analysis}

Data analysis was performed by an independent statistician using SPSS (Version 22; SPSS Inc., Chicago, IL). Univariate analysis of continuous variables were com

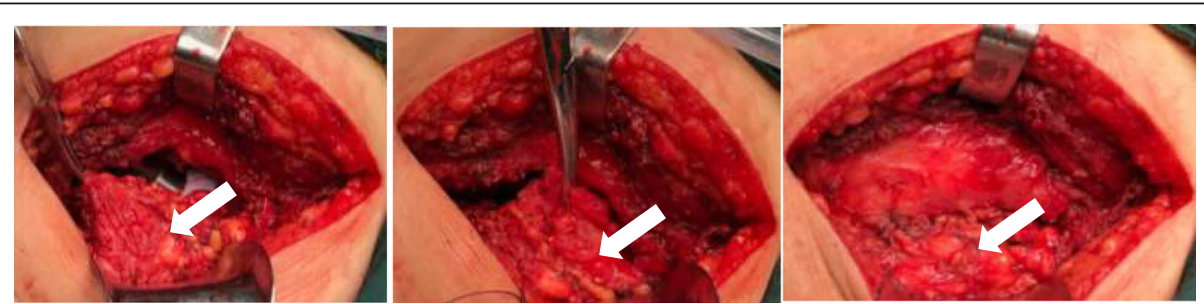

Fig. 2 a The flap-shaped incision of posterior joint capsule. $\mathbf{b}$ The detachment of piriformis tendon. $\mathbf{c}$ The non-in-situ reattachment of piriformis in combination with the posterior joint capsule on the posterior part of femoral great trochanter 

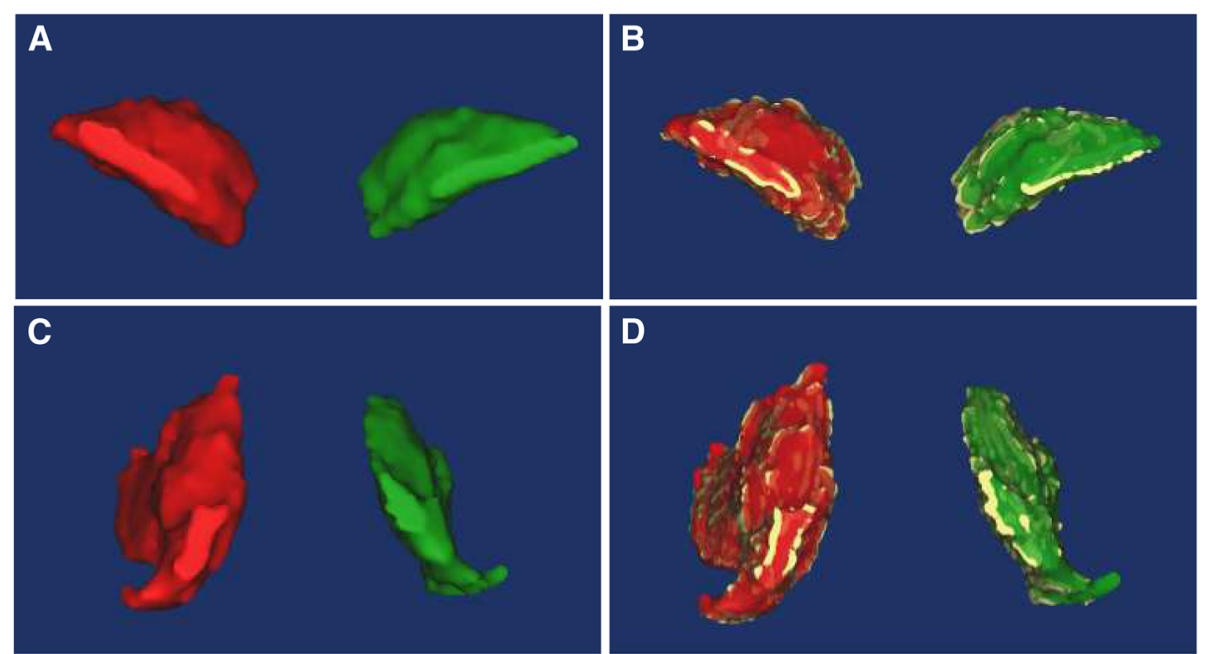

Fig. 3 a The typical three-dimensional MRI reconstruction of bilateral piriformis. $\mathbf{b}$ The typical three-dimensional MRI reconstruction of bilateral piriformis with adipose tissue. c The typical three-dimensional MRI reconstruction of bilateral internal obturator muscle. $\mathbf{d}$ The typical threedimensional MRI reconstruction of bilateral internal obturator muscle with adipose tissue. (The yellow represents adipose tissue)

pared with a parametric paired test or a nonparametric Mann-Whitney $U$ test. Because of the relatively small sample size, continuous variables were tested on normality using a Shapiro-Wilk test. When the $p$ value was less than 0.05 , data were assumed to not be normally distributed, and the nonparametric test was used. Since the study was self-paired design,

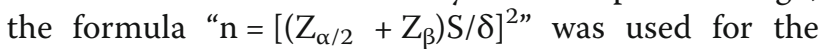
calculation of the sample size $(\alpha=0.05, \beta=0.1)$. A p value less than 0.05 was deemed to be statistic significant.

\section{Results}

External rotators morphology analysis by threedimensional MRI reconstruction

\section{External rotators volume}

There was not a significant difference regarding the muscle volume of the bilateral piriformis and internal obturator muscle preoperatively (piriformis: $p=0.085$; internal obturator muscle: $p=0.09$ ). At 6 weeks postoperatively, compared with the contralateral side, the muscle volume of piriformis on the operated side decreased by $1.64 \%$ ( $p=0.062$ ), while $7.28 \%$ for the internal obturator muscle $(p<0.01)$. At 12 weeks, the piriformis atrophy was still inapparent (decreased by $1.33 \%, p=$ 0.057); nevertheless, the muscle volume of internal obturator muscle continued to reduce (decreased by $15.71 \%, p<0.01)$. Up to 52 weeks, the pirformis also showed significant muscle atrophy, which decreased by $7.79 \%(p<0.01)$, and $24.18 \%$ for internal obturator muscle $(p<0.01)$ (Fig. 4). There was no significant change in the muscle volume on the contralateral side during the entire follow-up $(p>0.05)$.

\section{External rotators fat-muscle ratio}

No statistical significance (piriformis: $p=0.061$; internal obturator muscle: $p=0.067$ ) was found in the fat-muscle ratio of bilateral external rotators preoperatively. At 6 weeks postoperatively, the fat-muscle ratio of piriformis on the operated side only increased by $0.26 \%(p=0.071)$ in comparison with the contralateral side; however, the ratio of internal obturator muscle went up markedly (increased by $2.09 \%, p<0.01)$. At 12 weeks, the fatty infiltration degree of piriformis remained stable, which increased by $0.20 \%$; and the ever-increasing ratio of internal obturator muscle presented more significant (increased by $5.14 \%, p<0.01)$. At 52 weeks, both the piriformis and the internal obturator muscle manifested the dramatic augmentation in the fat-muscle ratio (piriformis: $4.21 \%, p<0.01$; internal obturator muscle: $11.91 \%, p<0.01$ ) (Fig. 5). There was no obvious change in the fat-muscle ratio on the contralateral side during the entire follow-up $(p>0.05)$.

\section{Clinical assessment \\ External rotation range}

The external rotation range of the bilateral hip joint was at the same baseline level preoperatively $(p=0.066)$, while it was limited obviously on the operated side at 6 weeks postoperatively (average of $36.75^{\circ}, p<0.01$ ). At 12 weeks, it got closed to the normal value, but the difference was still significant (average of $40.09^{\circ}, p<0.01$ ). After 52 weeks of rehabilitation, the external rotation angle completely recovered (average of $42.43^{\circ}, p=0.482$ ) (Fig. 6). There was no statistical significance in the hip external rotation range on the contralateral side during the entire follow-up $(p>0.05)$. 

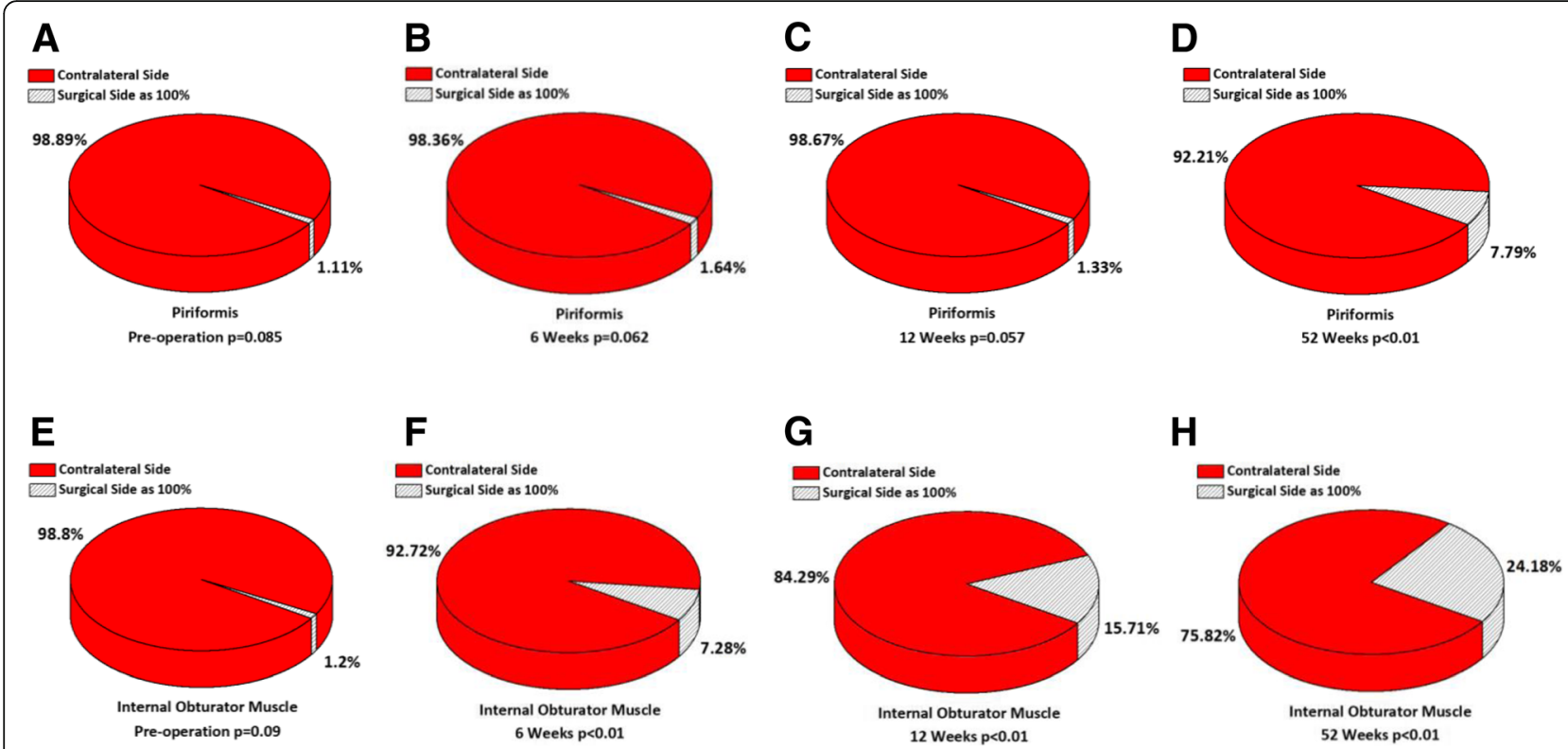

Fig. 4 The percentage of muscles volume on the operated side in comparison with the contralateral side from pre-operation to 52 weeks postoperatively. a-d The muscle volume of piriformis decreased by $1.11 \%(p=0.085)$ preoperatively, $1.64 \%(p=0.062)$ at 6 weeks, $1.33 \%(p=$ $0.057)$ at 12 weeks and $7.79 \%(p<0.01)$ at 52 weeks. e-h The muscle volume of internal obturator muscle decreased by $1.20 \%(p=0.09)$ preoperatively, $7.28 \%(p<0.01)$ at 6 weeks, $15.71 \%(p<0.01)$ at 12 weeks and $24.18 \%(p<0.01)$ at 52 weeks

\section{Discussion}

The posterior approach has been used most frequently among various THA approaches [10, 11]; it holds the advantages of clear anatomical layer, intact hip abductors, lower incidence of heterotopic ossification, and short learning cycle over the others. However, controversy still exists over the degree of approachrelated injury to the hip external rotators as well as whether to repair them or not. The results of this study proved more precisely that the posterior approach did seriously damage to the muscle mor phology of hip external rotators as well as the external rotation function. Moreover, the effective muscle repair was beneficial to the amelioration of the muscular morphological insufficiency.

Previous researches have already confirmed surgical injury to the external rotators via posterior THA [12-16]. Several pathologic researches have already identified that loss of tendon-bone adhesion could lead to muscle atrophy and fatty infiltration [17, 18]. In theory, there was no doubt that the detachment of the muscle tendon intraoperatively could cause the
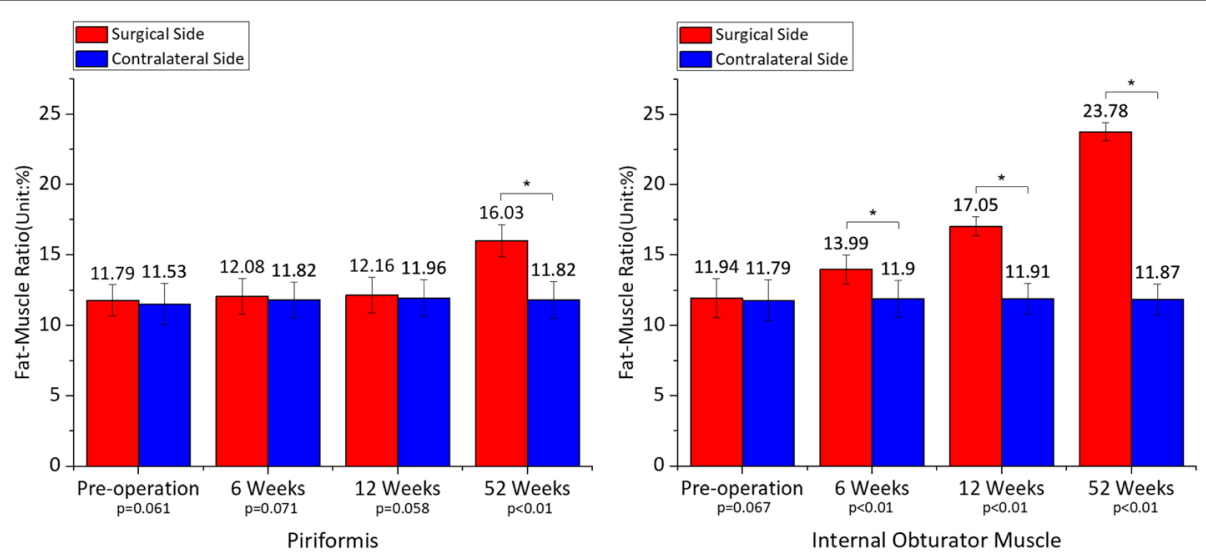

Fig. 5 Mean \pm standard deviation of fat-muscle ratio on the operated side in comparison with the contralateral side from pre-operation to 52 weeks postoperatively. a The fat-muscle ratio of piriformis increased by $0.26 \%(p=0.061)$ preoperatively, $0.26 \%(p=0.071)$ at 6 weeks, $0.20 \%(p=0.058)$ at 12 weeks and $4.21 \%(p<0.01)$ at 52 weeks. b The fat-muscle ratio of internal obturator muscle increased by $0.15 \%(p=0.067)$ preoperatively, $2.09 \%(p<$ $0.01)$ at 6 weeks, $5.14 \%(p<0.01)$ at 12 weeks and $11.91 \%(p<0.01)$ at 52 weeks 


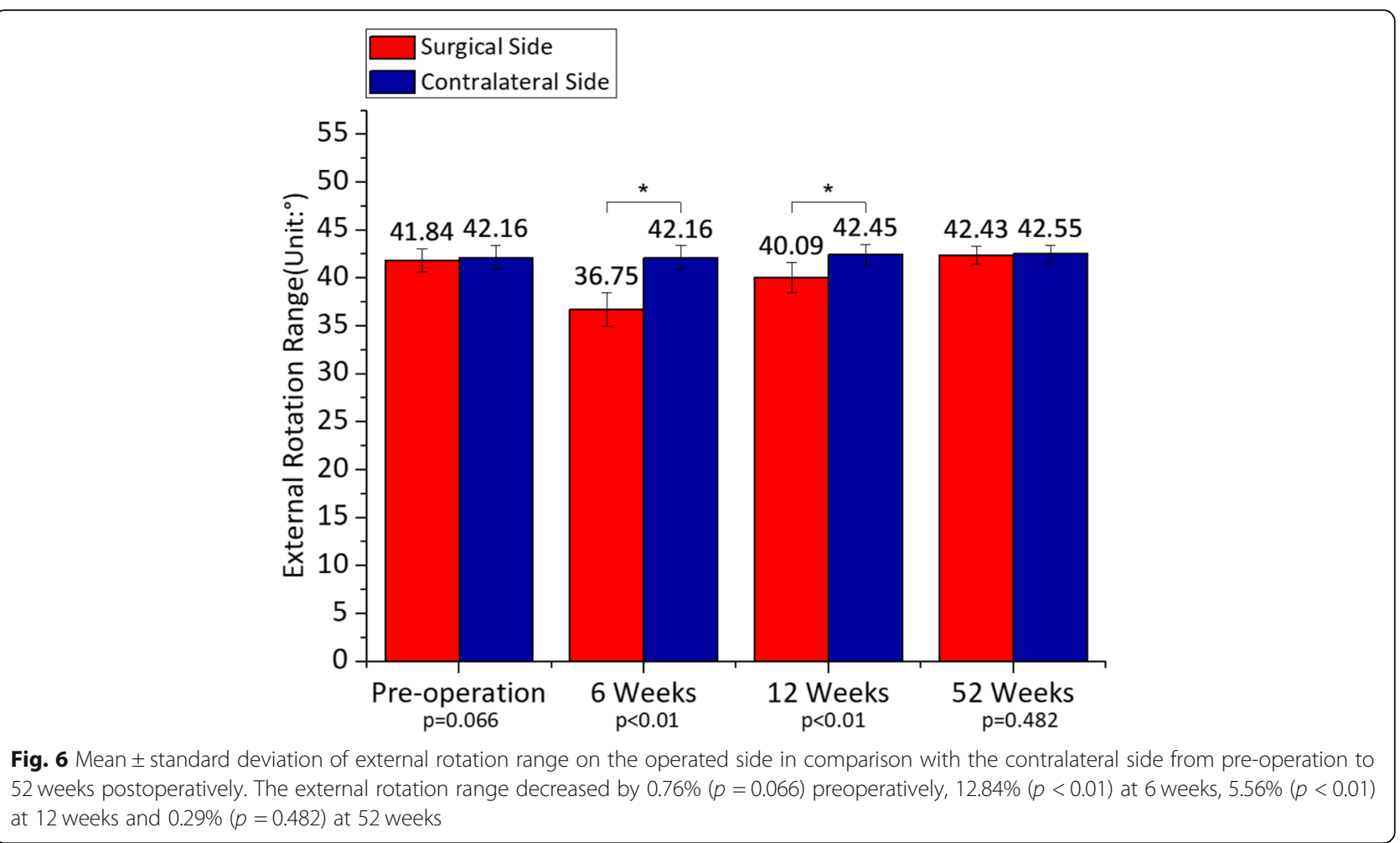

injury to the short external rotators. Still, the research conclusions without muscle morphology analysis could not be entirely convincing. MRI is currently the gold standard for analyzing muscle damage through morphological changes, mainly including the muscle volume atrophy and fatty infiltration [19-21]. Rarely, Khan RJ et al. reported significant deterioration in the muscle grade and nearly 50\% decrease in the muscle bulk of the piriformis via posterior approach through the two-dimensional MRI measurements [22]. Nevertheless, since two-dimensional measurement was limited to one or some specified MRI slices, a great loss of the key information of muscles was inevitable and then the results lacked accuracy and repeatability. Vidt ME et al. demonstrated that assessments of fatty infiltration and muscle atrophy from a single magnetic resonance image slice could not be predictive of 3-dimensional measurements [9]. In order to cover the shortage, our study took advantage of both accurate MRI morphology analysis and computer-assisted 3D reconstruction technique. Therefore, this study not only confirmed the previous research conclusions, but also further presented the extent of the muscle injury quantificationally. According to the authors' knowledge, the general idea and realization of the digital 3D-reconstruction of skeletal muscles based on MRI raw data is reported for the first time.

Muscle injury is inevitable, so how to minimize the injury seems more important. The previous studies related to the surgical injury of external rotators, whether to repair the muscles or not and the effect of repair provoked some other controversy. Researchers in favor of the repair claimed that it was necessary because the repaired muscles provided a physical scaffold-like support to stabilize the hip joint and decrease the early postoperative prosthetic dislocation rate [23-26]. Meanwhile, the more balanced hip joint motion as well as the better external rotation function were also mentioned [27]. Nevertheless, with a great risk for re-rupture and failure, the opponents against the posterior structure repair stood by the theory that the repaired posterior structure could not meet the strength of daily routine activity and some actually insisted that the repair itself was not responsible for the lower dislocation rate [28-30]. Undoubtedly, the morphology and the quality of the short external rotators directly determine the muscular function as well as the joint stability [31]. Nevertheless, few studies have focused on its changes whether did the repair or not. Consequently, this study quantificationally analysed the effect of the repair based on the recovery of the muscular morphology at the early postoperative period by the 3D-MRI reconstruction. In our research, the results demonstrated the more obvious degeneration of the incised but unrepaired muscle comparing to the repaired. In addition, as the anatomical characteristic of the short external rotators, the root of the internal obturator muscle is connected to the levator ani muscle which is closely involved in supporting the pelvic organs. 
Tomonori Baba et al. found that the symptoms of urinary incontinence were significantly aggravated after THA via the posterior approach due to the internal obturator muscle damage. If the tension and strength of the external rotators recovers, support of the pelvic organs and urinary incontinence may be improved [32]. Although there were no postoperative complications of prosthesis dislocation and urinary incontinence in this study, the muscle damage as well as the significant effects of the effective muscle repair was obvious. More interestingly, Tetsu Yamaguchi et al. found that the reconstruction of the short external rotators had significantly higher abduction muscle strength and external rotation muscle strength, and it could improve joint stability without limiting range of motion (ROM) [33]. Therefore, we recommend the reattachment of the detached external rotators if conditions permit. We believe that the repair provides a primary tendon insertion and probably does conduce to the early postoperative muscle recovery. It is also important to note that non-in-situ suture repair can provide initial stability for muscle recovery, but muscle degeneration will still occur slowly as time passed. Whereas, due to the muscle contracture caused by the chronic diseases, it is difficult for the external rotators to be repaired in situ. Moreover, in order to further explore the value of the repair, a extended analysis about the effects of posterior reconstruction on the important hip muscle groups has been in progress.

The main limitations of the study are listed below: 1 . the limited size of cohort and follow-up time; 2. Influence on the statistical power from patients lost during the follow-up. While there was not a significant difference on the baseline data of the lost and retained and the long-term effect of the repair needs to be further studied in a larger sample clinical trial.

\section{Conclusion}

The posterior THA caused serious damage to the external rotators and early postoperative external rotation function. An effective repair useful to the early postoperative recovery of external rotators in comparison with no repair management should be considered.

\section{Abbreviation}

EMG: electromyography; ROM: range of motion; THA: total hip arthroplasty

\section{Acknowledgements}

Not applicable.

Funding

No specific funding was received.

\section{Availability of data and materials}

All data is stored in the clinical trial registry. And the datasets used or analysed during the current study are available from the corresponding author on reasonable request.

\section{Ethical review committee statement}

This prospective study has been approved by Ethics Committee of the First Affiliated Hospital of Chongqing Medical University. Meanwhile, the study has been registered in Chinese Clinical Trial Registry (ChiCTR) before the clical trial started, the Clinical Trial Registry Number is ChiCTR-IOR-17013007.

\section{Authors' contributions}

Conceptualization: TW, WH, LS, WX, FLL. Data curation: TW, LS. Formal analysis: TW, LS, WX, FLL. Investigation and measurement: TW, LS, WX. Methodology: TW, LS, WH, WX. Project administration: TW, LS, WH, WX. Writing-original draft: TW, LS, WX, FLL. Writing-review\&editing: TW, WH, LS, WX. Each author have participated sufficiently in the work to take public responsibility for appropriate portions of the content, and agreed to be accountable for all aspects of the work in ensuring that questions related to the accuracy or integrity of any part of the work are appropriately investigated and resolved. TW and LS contributed equally. All authors read and approved the final manuscript.

Ethics approval and consent to participate

Ethical review committee statement: This prospective study has been approved by Ethics Committee of the First Affiliated Hospital of Chongaing Medical University and registered in Chinese Clinical Trial Registry (ChiCTR).

\section{Consent for publication}

N/A

Competing interests

The authors declare that they have no competing interests.

\section{Publisher's Note}

Springer Nature remains neutral with regard to jurisdictional claims in published maps and institutional affiliations.

Received: 19 July 2018 Accepted: 3 December 2018

Published online: 14 January 2019

\section{References}

1. Waddell J, Johnson K, Hein W, Raabe J, FitzGerald G, Turibio F. Orthopaedic practice in total hip arthroplasty and total knee arthroplasty: results from the global Orthopaedic registry (GLORY). Am J Orthop (Belle Mead NJ). 2010:39:5-13.

2. Gibson A. Posterior exposure of the hip joint. J Bone Joint Surg Br. 1950;32: $183-6$.

3. Maratt JD, Gagnier JJ, Butler PD, Hallstrom BR, Urquhart AG, Roberts KC. No difference in dislocation seen in anterior vs posterior approach Total hip arthroplasty. J Arthroplast. 2016;31:127-30

4. Cohen RG, Katz JA, Skrepnik NV. The relationship between skeletal muscle serum markers and primary THA. Clin Orthop Relat Res. 2006;467:1747-52.

5. Michael Meneghini R, Smits SA. Rachel R. Swinford and Rafael E. Bahamonde. A randomized, prospective study of 3 minimally invasive surgical approaches in Total hip arthroplasty-comprehensive gait analysis. J Arthroplast. 2008:23:68-73.

6. Meneghini RM, Pagnano MW, Trousdale RT, Hozack WJ. Muscle damage during MIS total hip arthroplasty: Smith-Petersen versus posterior approach. Clin Orthop Relat Res. 2006;453:293-8.

7. McLawhorn AS, Potter HG, Cross MB, Boettner F, Lim W, Lee YY, Pellicci PM. Posterior soft tissue repair after primary THA is durable at mid-term Followup: a prospective MRI study. Clin Orthop Relat Res. 2015:473:3183-9.

8. Müller M, Tohtz S, Dewey M, Springer I, Perka C. Age-related appearance of muscle trauma in primary total hip arthroplasty and the benefit of a minimally invasive approach for patients older than 70 years. Int Orthop. 2001:35:165-71.

9. Vidt ME, Santago AC II, Tuohy CJ, Poehling GG, Freehill MT, Kraft RA, Marsh AP, Hegedus EJ, Miller ME, Saul KR. Assessments of fatty infiltration and muscle atrophy from a single magnetic resonance image slice are not predictive of 3-dimensional measurements. J Arthrosc Relat Surg. 2016;32: 128-39.

10. Puolakka TJS, Pajamäki KJ, Halonen PJ, Pulkkinen PO, Paavolainen P, Nevalainen JK. The Finnish arthroplasty register-report of the hip register. Acta Orthop Scand. 2001;72:433-41. 
11. Sadoghi P, Leithner A, Labek G. Overcoming boundaries of worldwide joint arthroplasty registers: the European arthroplasty register minimal dataset. J Arthroplast. 2013;28:1327-8.

12. Agten CA, Sutter R, Dora C, Pfirrmann CW. MR imaging of soft tissue alterations after total hip arthroplasty: comparison of classic surgical approaches. Eur Radiol. 2017;27:1312-21.

13. Goutallier D, Postel JM, Bernageau J, Lavau L, Voisin MC. Fatty muscle degeneration in cuff ruptures. Pre- and postoperative evaluation by $\mathrm{CT}$ scan Clin Orthop Relat Res. 1994;(304):78-83.

14. Bergin PF, Doppelt JD, Kephart CJ, Benke MT, Graeter JH, Holmes AS, Haleem-Smith H, Tuan RS, Unger AS. Comparison of minimally invasive direct anterior versus posterior total hip arthroplasty based on inflammation and muscle damage markers. J Bone Joint Surg Am. 2011;93:1392-8.

15. White J, Kelly M, Dunsmuir R. C-reactive protein level after total hip and total knee replacement. J Bone Joint Surg Br. 1998;80:909-11.

16. Borg H, Kiviranta I, Anttila E, Häkkinen K, Ylinen J, Kautiainen H, Häkkinen A. External rotation strength deficit after hip resurfacing surgery. Disabil Rehabil. 2009:31:865-70.

17. Ruoss S, Möhl CB, Benn MC, von Rechenberg B, Wieser K, Meyer DC, Gerber C, Flück M. Costamere protein expression and tissue composition of rotator cuff muscle after tendon release in sheep. J Orthop Res. 2018;36:272-81.

18. Akimoto T, Ushida T, Miyaki S, Akaogi H, Tsuchiya K, Yan Z, Williams RS, Tateishi T. Mechanical stretch inhibits myoblast-to-adipocyte differentiation through Wnt signaling. Biochem Biophys Res Commun. 2005;329:381-5.

19. Müller M, Tohtz S, Dewey M, Springer I, Perka C. Muscle trauma in primary total hip arthroplasty depending on age, BMl, and surgical approach. Minimally invasive anterolateral versus modified direct lateral approach. Orthopade. 2011:40:217-23.

20. Müller M, Tohtz S, Dewey M, Springer I, Perka C. Evidence of reduced muscle trauma through a minimally invasive anterolateral approach by means of MRI. Clin Orthop Relat Res. 2010;468:3192-200.

21. Vasilakis I, Solomou E, Vitsas V, Fennema P, Korovessis P, Siamblis DK. Correlative analysis of MRI-evident abductor hip muscle degeneration and power after minimally invasive versus conventional unilateral Cementless THA. Orthopedics. 2012;35:e1684-91.

22. Khan RJ, Lam LO, Breidahl W, Blakeney WG. Magnetic resonance imaging features of preserved vs divided and repaired piriformis during Total hip arthroplasty. J Arthroplast. 2012;27:551-8.

23. Browne JA, Pagnano MW. Surgical technique: a simple soft-tissue-only repair of the capsule and external rotators in posterior-approach THA. Clin Orthop Relat Res. 2012;470:511-5.

24. Prietzel T, Hammer N, Schleifenbaum S, Adler D, Pretzsch M, Köhler L, Petermann M, Farag M, Panzert S, Bauer S, von Salis-Soglio G. The impact of capsular repair on the dislocation rate after primary total hip arthroplasty: a retrospective analysis of 1972 cases. Z Orthop Unfall. 2014;152:130-43.

25. Zhang Y, Tang Y, Zhang C, Zhao X, Xie Y, Xu S. Modified posterior soft tissue repair for the prevention of early postoperative dislocation in total hip arthroplasty. Int Orthop. 2013;37:1039-44.

26. Pellicci PM, Bostrom M, Poss R. Posterior approach to total hip replacement using enhanced posterior soft tissue repair. Clin Orthop Relat Res. 1998;355: 224-8.

27. Mihalko WM, Whiteside LA. Hip mechanics after posterior structure repair in total hip arthroplasty. Clin Orthop Relat Res. 2004;420:194-8.

28. Kao JT, Woolson ST. Piriformis tendon repair failure after total hip replacement. Orthop Rev. 1992;21:171-4.

29. Loiba V, Stucinskas J, Robertsson O, Wingstrand H, Tarasevicius S. The analysis of posterior soft tissue repair durability after total hip arthroplasty in primary osteoarthritis patients. Hip Int. 2015;25:420-3.

30. Stähelin T, Vienne P, Hersche O. Failure of reinserted short external rotator muscles after total hip arthroplasty. J Arthroplast. 2002;17:604-7.

31. Grimaldi A. Assessing lateral stability of the hip and pelvis. Man Ther. 2011; 16:26-32.

32. Baba T, Homma Y, Takazawa N, Kobayashi H, Matsumoto M, Aritomi K, Yuasa T, Kaneko K. Is urinary incontinence the hidden secret complications after total hip arthroplasty? Eur J Orthop Surg Traumatol. 2014;24:1455-60.

33. Yamaguchi T, Naito M, Asayama I, Kambe T, Fujisawa M, Ishiko T. The effect of posterolateral reconstruction on range of motion and muscle strength in Total hip arthroplasty. J Arthroplast. 2003;18:347-51.

\section{Ready to submit your research? Choose BMC and benefit from:}

- fast, convenient online submission

- thorough peer review by experienced researchers in your field

- rapid publication on acceptance

- support for research data, including large and complex data types

- gold Open Access which fosters wider collaboration and increased citations

- maximum visibility for your research: over $100 \mathrm{M}$ website views per year

At BMC, research is always in progress.

Learn more biomedcentral.com/submissions 